\title{
Research on Multi-Stage Generation and Transmission Coordinated Plan- ning Model Considering Wind Power Integration
}

\author{
Zeng Ming, Ouyang Shaojie, Shi Hui ${ }^{*}$ and Ge Yujian
}

School of Economics and Management, North China Electric Power University, Beijing, 102206, China

\begin{abstract}
Decentralized planning mode of power generation and power transmission is facing enormous challenges at present, and all kinds of uncertainties that impact on the planning have become increasingly evident. To solve the two coordinated planning and development issues is imminent, proposing a coordinated planning model of generation and transmission to improve system stability and flexibility and reduce the uncertainties whose threat is significant. Combined with the Stackelberg game theory, this proposes a two-stage coordinated planning model of generation and transmission, which can effectively provide decision makers with optimal program of investment decision, and control the investment risk of power system construction. And the this paper uses the Heuristic Artificial Fish Swarm Algorithm (HAFSA) to solve this model and illustrates our results using a 24-bus network example.
\end{abstract}

Keywords: Coordinated planning, heuristic artificial fish swarm algorithm, Stackelberg game, wind power integration.

\section{INTRODUCTION}

Nowadays, the accelerated pace of smart grid construction, the increasing demand of cross-regional electricity, the grid connection of wind and other renewable energy, and the confirmation of the targets of energy conservation and emissions reduction make the planning of power generation and power transmission face more kinds of uncertainties. And they also make the requirement on coordination between power generation and power transmission become higher. So, proposing a coordinated planning model of generation and transmission to improve stability and flexibility of the system and reduce the uncertainties threat is significant for the orderly development of modern electric power system.

In the process of power generation and transmission planning, there exists many uncertainties, such as the coordination of planning, the system robustness and so on [1]. But until now, most of the power transmission planners use deterministic or single-stage planning model of transmission to plan on transmission [1]. There are some shortcomings in these models; for example, they are too fragile and highly sensitive to assumption. In paper [2], based on Stackelberg game theory, a three-stage game model has been proposed in which power transporters are regarded as leaders, but it does not consider the necessity for adjusting the planning of power transmission to adapt to development in the future. Others have also proposed the interaction between power generation and power transmission in open market, but they only considered the case of certainty or load changes per hour [3].

*Address correspondence to this author at the Changping District Beinong Road, Beijing, China. 102206; Tel: 15810296098;

E-mail: 953662383@qq.com
This paper considers the wind power paralleling in the grid and the case of Stackelberg game in the coordinated planning of generation and transmission. And it aims to solve the problem of the instability of wind power paralleling in the grid and the poor coordinated planning of generation and transmission. The capacity constraints, trends constraints, regional power constraints have been considered, and various uncertain factors have been introduced. Then this paper proposes a multi-stage coordinate planning model of generation and transmission, which aims to maximize the final social welfare expenditure. Last, it uses the Heuristic Artificial Fish Swarm Algorithm(HAFSA) to solve this model and illustrates the results using a 24-bus network example.

\section{MODEL CONSTRUCTION}

\subsection{Objective Function}

We assume that the electric power market is perfectly competitive, where there are $N$ generation companies in each bus. Each generation company has different ways of power generating $g$. And in each generation company, a certain way of power generation is achieved to output by one unit. This model can meet the trends constraints, capacity constraints, Kirchhoff's law and climbing constraints, and achieve the maximization of social welfare. Based on Stackelberg game theory, the coordinated planning process of generation and transmission has been divided into three stages $h(h=1,2,3)$, and main objective function has been derived by reverse method.

The third stage is the power market operation stage, and the maximization of benefits of single generation company $\mathrm{c}$ $(\mathrm{c} \in \mathrm{C})$ is as follows: 
$\max _{\left\{q_{i, g}^{s}, i \in I, g \in G\right\}} \pi_{c}^{s}=\sum_{g \in G} \sum_{i \in I}\left\{P_{i, g}^{s}\left(q_{i, g}^{s}+r_{i}^{s}\right) \cdot q_{i, g}^{s}\right.$

$\left.-C P_{i, g}^{s}\left(q_{i, g}^{s}, p_{i, g}^{s}\right)\right\}$

s.t. $q_{i, g}^{s} \geq 0, i \in I, g \in G$

Where, $S$ represents the set of coordinated planning scenarios of generation and transmission, $S=\left\{s / s=s_{n}, \quad n=1,2,3\right\}$, $s_{n}$ represents the $n$th planning scenario; $I$ represents the set of buses, $I=\left\{i / i=i_{n}, n=1,2,3 \ldots\right\}, i_{n}$ represents the $n$ th-bus; $G$ represents the set of generation ways, $G=\left\{g / g=g_{n}, n=1,2,3 \ldots\right\}$, $g_{n}$ represents the $n$th generation way; $C$ represents the set of generation companies, $C=\left\{c / c=c_{n}, n=1,2,3 \ldots\right\}, c_{n}$ represents the nth generation company; qs $i, g$ represents the generated energy of generation way $\mathrm{g}$ in scenario $s$ and in $i$ thbus; $r s i$ represents the input power in scenario $s$ and in $i$ thbus; $P s i, g(\cdot)$ represents the inverse function of demand function of generation way $g$ in scenario $s$ and in $i$ th-bus; $C P s$ $i, g(q s i, g, p s i, g)$ represents the production cost function of generation way $g$ in scenario $s$ and in $i$ th-bus; $p s i, g$ represents generating capacity of generation way $g$ in scenario $s$ and in $i$ th-bus. lows.

On this basis, the maximization of grid revenue is as fol-

$$
\max _{y_{i}} \sum_{i=1}^{I} w_{i} \cdot y_{i}
$$

s.t. $-T_{k} \leq \sum_{i=1}^{I} D_{k i} \cdot y_{i} \leq T_{k}, \forall k$

Where, $w_{i}$ represents the price of power transmission from transaction center to $i$ th-bus; $y_{i}$ represents the transmission capacity from transaction center to $i$ th-bus; $\pm T_{k}$ represents the upper and lower conveying capacity of electric transmission line $k ; K$ represents the set of electric transmission line, $K=\left\{k / k=k_{n}, \quad n=1,2,3 \ldots\right\}, k_{n}$ represents the $n$th electric transmission line; $D_{k i}$ represents power transfer distribution factor from electric transmission line $k$ to $i$ th-bus. $\sum D_{k i} \cdot y_{i}$ represents the net power through electric transmission line $k$.

KKT(Karush-Kuhn-Tucker) constraints of (1) and (2) are as follows:

$$
\left\{\begin{array}{c}
\frac{\partial P_{i, g}^{s}\left(q_{i, g}^{s}+r_{i, g}^{s}\right)}{\partial q_{i, g}^{s}} \cdot q_{i, g}^{s}+P_{i, g}^{s}\left(q_{i, g}^{s}+r_{i, g}^{s}\right)- \\
\frac{\partial C P_{i, g}^{s}\left(q_{i, g}^{s}, g_{i, g}^{s}\right)}{\partial q_{i, g}^{s}}+\lambda_{i}^{s}=0, \\
\forall i \in I, c \in C, s \in S \\
\lambda_{i}^{s} \cdot q_{i, g}^{s}=0, \quad \forall i \in I, c \in C, s \in S \\
q_{i, g}^{s} \geq 0, \quad \forall i \in I, c \in C, s \in S \\
\lambda_{i}^{s} \geq 0, \quad \forall i \in I, c \in C, s \in S
\end{array}\right.
$$

Where, $\lambda s$ iis Lagrange multiplier of (1) and (2).
KKT(Karush-Kuhn-Tucker) constraints of (3) and (4) are as follows.

$$
\left\{\begin{array}{l}
w_{i}+\sum_{k} D_{k i}\left(-\lambda_{k 1}+\lambda_{k 2}\right)=0 \\
T_{k}-\sum_{i=1}^{I} D_{k i} \cdot y_{i} \geq 0, \lambda_{k 1} \geq 0 \\
\left(T_{k}-\sum_{i=1}^{I} D_{k i} \cdot y_{i}\right) \cdot \lambda_{k 1}=0 \\
T_{k}+\sum_{i=1}^{I} D_{k i} \cdot y_{i} \geq 0, \lambda_{k 2} \geq 0 \\
\left(\begin{array}{c}
T_{k}-\sum_{i=1}^{I} D_{k i} \cdot y_{i}
\end{array}\right) \cdot \lambda_{k 2}=0
\end{array}\right.
$$

The second stage is the phase in which generation companies make investment and expansion capacity decisions. Different expansion capacity programs have decisive effect on investment returns of generation companies. Generation company $c$ achieves the maximization of investment returns by (7):

$$
\begin{aligned}
& \max _{\left\{p_{i, g}, i \in I\right\}} E_{s}\left[\pi_{c}^{s}\right]-\sum_{i \in I, g \in G}\left\{C I G_{i, g}\left(p_{i, g}, p_{i, g}^{0}\right)\right\} \\
& \text { s.t. (5)-(6) }
\end{aligned}
$$

Where, $E_{s}[\pi]$ represents expected income; $p 0 i, g$ represents generating capacity of generation way $g$ which generation company $c$ owes in initial stage and in $i$ th-bus; $p_{i, g}$ represents generating capacity of generation way $g$ which generation company $c$ owes in $i$ th-bus; $C I G_{i, g}\left(p_{i, g}, p 0 i, g\right)$ represents capitalized cost which generation company $c$ needs for expansion capacity of generation way $g$ in $i$ th-bus.

The first stage is the phase in which the planners make investment decisions of the planning of transmission. By assessing the different transmission investment programs and predicting the possible expansion capacity, decisions are made by transmission planners. The main objective function is as follows:

$$
\begin{aligned}
& \max _{k, f_{k}} \sum_{i \in I}\left\{E_{s}\left[\int_{0}^{q_{i, g}^{s}+r_{i}^{s}} P_{i, g}^{s}(q) d q-C P_{i, g}^{s}\left(q_{i, g}^{s}, p_{i, g}^{s}\right)\right]\right. \\
& \left.-C I G_{i, g}\left(p_{i, g}, p_{i, g}^{0}\right)\right\}-C I_{k}\left(f_{k}, f_{k}^{0}\right) \\
& \text { s.t. (5)-(6) }
\end{aligned}
$$

Where, $C I_{k}\left(f_{k} f \circ k\right)$ represents the capitalized cost of expansion capacity of electric transmission line $k$ in the first stage; $f_{k}$ represents the transmission capacity constraints after expanding capacity of electric transmission line $k$ in the first stage; $f 0 k$ represents the transmission capacity constraints before expanding capacity of electric transmission line $k$ in the first stage.

\subsection{Constraint Conditions}

This paper ignores the influence of transmission loss. And the constraint conditions of the planning model include 


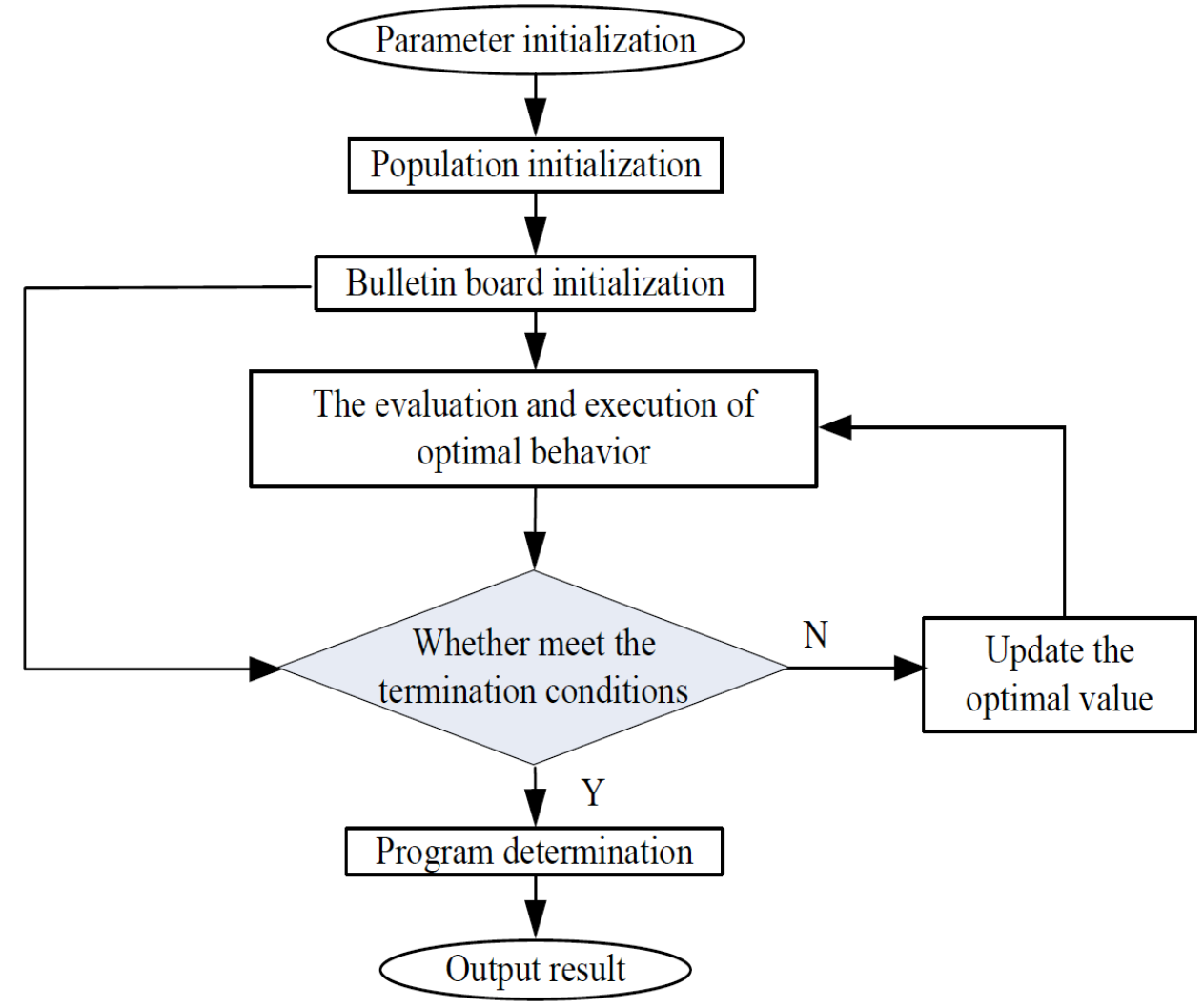

Fig. (1). Solution procedure of transmission planning model.

wind generation proportion constraints, power supply and demand balance constraints, generating capacity constraints, trend constraints, conventional power generation constraints and so on. The constraints related to wind power refer to paper [4]. The constraints related to conventional generating units refer to paper [5]. And other constraints refer to paper [6-9].

\section{MODEL SOLUTIONS}

This paper uses HAFSA to solve the proposed model. Compared with the entropy law, tradition artificial fish swarm algorithm, simulated annealing algorithm and other modern optimization algorithms, HAFSA has the characteristics of strong search capabilities, fast convergence and high accuracy.

HAFSA has three main behaviors: "evolution" prey behavior, "experience" swarm behavior and "experience" follow behavior. (1) "evolution" prey behavior(AF-prey): it is assumed that $X_{i}$ is the current state of artificial fishes, and searching $X_{i}$ in $\mathrm{M}$ times, then getting a new state $X_{\text {nest }}$. (2)"experience" swarm behavior(AF-swarm): it is assumed that $X_{i}$ is the current state of artificial fishes, $X_{c}$ is the center of the partners. If the forward conditions are met, the artificial fishes will step forward to $X_{c}$ and $X_{\text {retain }}$. Otherwise, it will execute the "evolution" prey behavior. (3)"experience" follow behavior(AF-follow): it is assumed that $X_{i}$ is the current state of artificial fishes, and searching for optimal partner within a certain range, if the forward conditions are met, the artificial fishes will step forward to $X_{c}$ and $X_{\text {retain }}$. Otherwise, it will execute the "evolution" prey behavior. The flow diagram for using HAFSA to solve the two-stage planning model of generation and transmission is shown in Fig. (1):

(1) Initialization Parameter: fishes scale $N_{s}$, maximum mobile step length Length, the perceive range of artificial fishes Range, crowded degree factor $\delta$, maximum search times for prey $M$ and so on. At last, combined with the Stackelberg game theory, the new multi-stage coordinated planning model of generation and transmission with wind power integration is established.

(2) Population initialization: put $N_{s}$ artificial fishes into the feasible region, and the set of execute solution in different scenarios is established.

(3) Bulletin board initialization: input the state of optimal fish $X_{b s e t}$ and function group to bulletin board, then judge whether it meets the termination conditions, if it achieves the maximization of main objective function (7), turn to step 7, or turn to step 4.

(4) Evaluate the "experience" swarm behavior, follow behavior of each artificial fish, search for the local optimization, and select the optimal behavior to execute. If missing, execute the "evolution" prey behavior. 


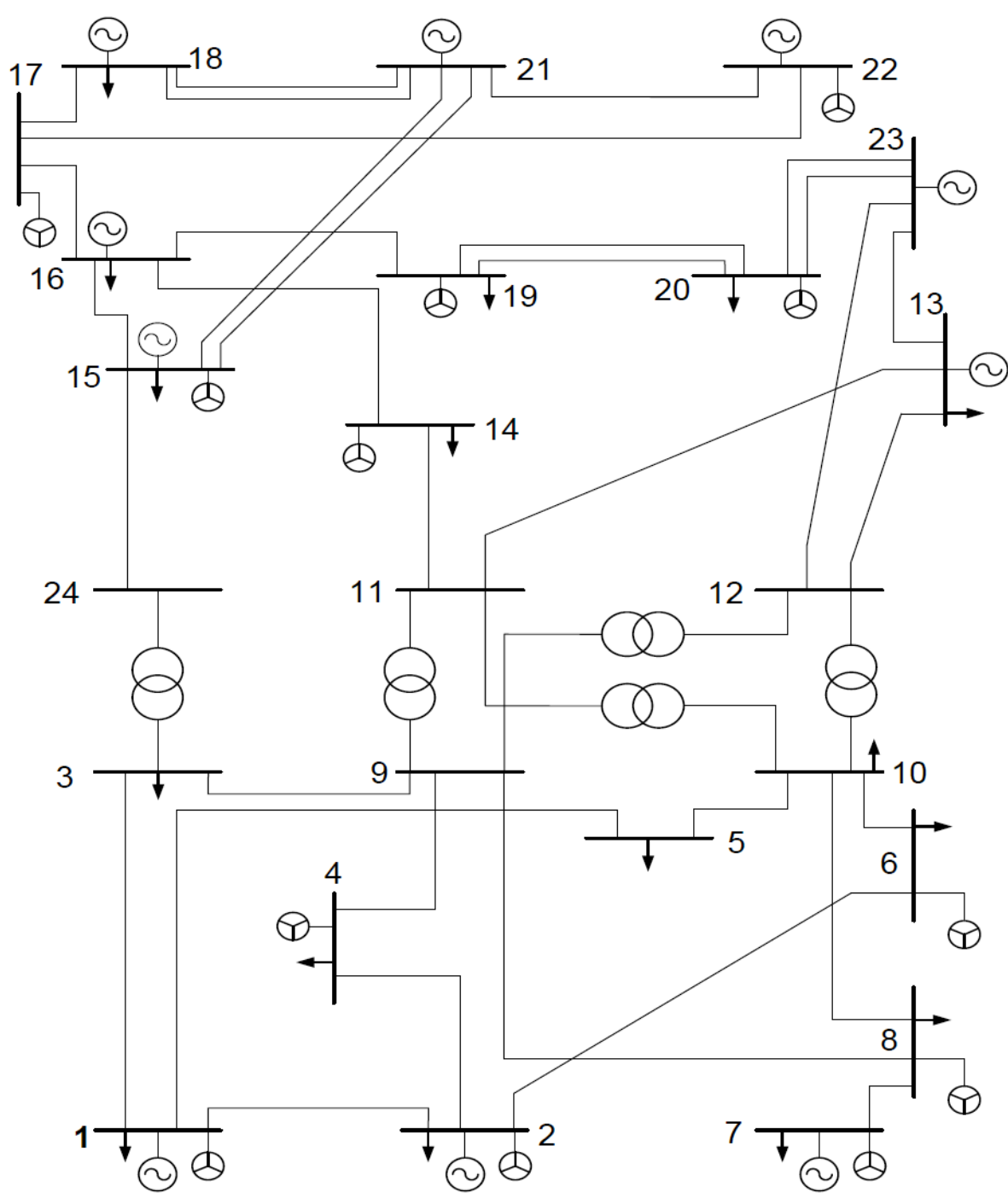

Fig. (2). IEEE-24 bus test system.

(5) Update the bulletin board: evaluate the new state of artificial fishes after step 4, if the result is superior to the bulletin boards, update the bulletin board. Meanwhile judge whether it meets the termination conditions.

(6) Update the optimal value: use the state of optimal fish to replace the state of worst fish in new shoal, and record the local optimal planning program as the global optimal planning program, then turn to step 4.

(7) Terminate the computation, and output the optimal coordination planning program and the maximum value of social welfare.

Above the flow diagram, we can see that step 4, step 5 and step 6 are the key steps in the process of algorithm iteration. By searching the better program, the whole algorithm phases out the sub-optimal program. In this way, it can achieve rapid convergence to get the effect of optimal program. The concrete solution process is shown in [7].

\section{EXAMPLE AND RESULT}

The paper uses improved IEEE-24bus system to analyze the multi-stage coordinated planning problem of generation and transmission with wind power integration [6]. The system structure is shown in Fig. (2) (in Fig. 2, power supply points to the sign of wind power present in the access points of wind power). There are 24 independent buses in the system, and 12 of these buses are accessed to wind power. Considering the future load demand, the changes in the cost of power generation and transmission and the large scale of wind power paralleling in the grid, the purpose of the model optimization is to ensure the coordination and consistency of the planning of generation and transmission, and achieve the maximization of social welfare.

The system initializes the data of generating capacity and load demand. The transmission line parameters and investment costs refer to $[8,9]$ (If the same parameter is 
Table 1. Operation feature for different forms of electricity generation.

\begin{tabular}{|c|c|c|c|c|c|}
\hline $\begin{array}{c}\text { Power Generation } \\
\text { Way }\end{array}$ & $\begin{array}{c}\text { Power Generation } \\
\text { Investment Cost / }\end{array}$ & Variable Cost / \$ & $\begin{array}{c}\text { The maximum of } \\
\text { Single Capacity/MW }\end{array}$ & $\begin{array}{c}\text { Forced Outage } \\
\text { Rate/\% }\end{array}$ & $\begin{array}{c}\text { Planned Outage } \\
\text { Rate/\% }\end{array}$ \\
\hline \hline Thermal power & 658 & 22.33 & 150 & 15 & 11.2 \\
\hline Hydropower & 2843 & 1.72 & 300 & 5 & 8.6 \\
\hline Wind power & 940 & 0 & 5 & 1.8 & 1.2 \\
\hline
\end{tabular}

Table 2. Number of generators invested to build.

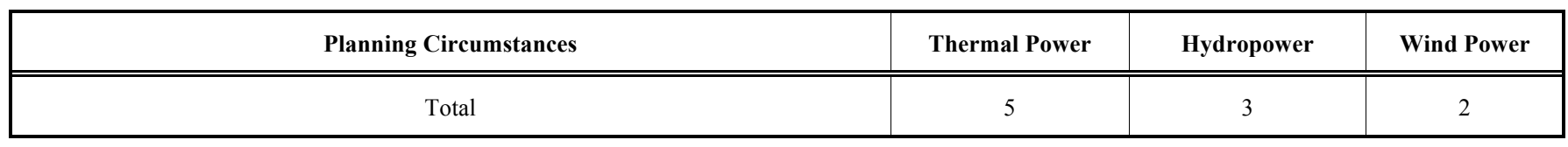

Table 3. Number of electric transmission line invested to build.

\begin{tabular}{|c|c|c|c|c|c|c|c|c|c|c|}
\hline First Bus To End Bus & $1-5$ & $3-24$ & $4-9$ & $6-10$ & $7-8$ & $10-11$ & $11-13$ & $14-16$ & $15-24$ & $16-17$ \\
\hline Total & 1 & 1 & 1 & 2 & 2 & 1 & 1 & 1 & 1 & 1 \\
\hline
\end{tabular}

inconsistent, conformed to [9]). The data of spare capacity rate and climbing rate refer to $[10,11]$, unit operation parameters are shown in Table $\mathbf{1}$. It is assumed that the parameters of the same type of units which each generation company has, are not different. For the investment decision of transmission and power generation in the first stage and the second stage, three scenarios can be considered: scenario 1 , scenario 2, scenario 3. Three scenarios are the events of equal probability. The data of Scenario assumption for coordination planning at stage one refers to [8-11], and the data of Scenario assumption for coordination planning at stage one refer to the data of the end of the first stage. Traditional planning method refers to [12].

The paper uses HAFSA to solve the multi-stage coordinate planning model of generation and transmission with wind power integration. And the results of coordinated planning are shown in Table $\mathbf{2}$ and Table $\mathbf{3}$.

Total social welfare expenditure calculated by the singlestage decentralized planning mode, which is not combined with Stackelberg game theory, is 343 million dollars. And the Total social welfare expenditure calculated by the method proposed in this paper is 403 million dollars. It is obvious that the coordinated planning model proposed increases the total social welfare expenditure by 60 million dollars. Thus, it can be seen that compared with the traditional planning model of power generation and power transmission, multistage coordinated planning model of generation and transmission which is combined with Stackelberg game theory, can improve the coordination and consistency of the planning of generation and transmission, reduce the unnecessary cost, enhance the overall economic benefits of the planning of generation and transmission, and effectively improve the total social welfare.

\section{CONCLUSION}

This paper comprehensively analyzes the coordinated planning problem of power generation and power transmission with wind power integration. Numerical results show: the multi-stage coordinated planning model of generation and transmission can effectively guarantee the consistency of the planning of generation and transmission, and improve the total social welfare expenditure. So this paper has a certain theoretical guiding significance to deal with problems in the process of power system planning, such as wind power layout, ratio of wind power integration and so on. But as previously mentioned, this paper ignores the influence of transmission loss. And there are also assumptions in building the model to simplify the uncertain factors. So in the future research work, further study is needed.

\section{CONFLICT OF INTEREST}

The authors confirm that this article content has no conflict of interest.

\section{ACKNOWLEDGEMENTS}

This paper belongs to the project of the National Science Foundation of China (NSFC) (71271082), The National Soft Science Research Program (2012GXS4B064) and the Energy Foundation of the U.S. (G-1006-12630).

\section{REFERENCES}

[1] Z. Ming, T. Kuo, X. Song, Q. Liu-qing, and D. Jun "Chance constrained transmission planning method based on chaos quantum honey bee algorithm," Power Syst. Protect. Control, vol. 38, no. 22 , pp. $1-7,2010$. 
[2] P. Vazquez, and F. Olsina, "Valuing flexibility of DG investments in transmission expansion planning," In: Proc. IEEE Power Tech., pp. 695-700, 2007.

[3] S. K. K. Ng, C. W. Lee, and J. Zhong, "A game-theoretic approach to study strategic interaction between transmission and generation expansion planning," In: Proc. 38th North Am. Power Sympos., pp. 115-120, 2006

[4] H. T. Wang, X. F. Liu, Z. H. Yun, H. D. Sun, and D. Z. Chen, "Two-tier and multi-stage voltage coordination control method for regional power grid with wind farms," Power Syst. Protect. Control, vol. 40, no. 22, pp. 1-13, 2012.

[5] Y. Bai, Y. Wang, Q. Xia, X. Sun, M. H. Yang, and J. Zhang, "A Full-scenario SCED with Coordinative Optimization of Hydrothermal-wind Power," In: Proc. of the CSEE, vol. 33, no. 13, pp. 29, 2013

[6] L. Chunquan, T. Kuo, and W. Yang, "Generation and Transmission Multi-Stage Coordination Planning Model Considering the Renewable Energy Integration", ECEPGC, vol. 41, no. 9, pp. 1814-1820, 2013.
[7] Q. Liangdong, H. Dengxu, and H. Yong, "Novel Heuristic Artificial Fish Swarm Algorithm," Comput. Eng., vol. 37, no.17, pp. 140-142, 2011

[8] M. Zeng, C. Lv, L. Qiu, and T. Kuo, "A Demand-Side ResponseBased Transmission Planning Model With Grid-Connected Wind Farms," Power Sys. Technol., vol. 35, no. 4, pp. 129-134, 2011.

[9] R. Romero, C. Rocha, and J. R. S. Mantovani, "Constructive heuristic algorithm for the DC model in network transmission expansion planning," In: IEEE Proc. Generation, transmission and Distribution, vol. 152, no. 2, pp. 277-282, 2005.

[10] B. F. Hobbs, G. Drayton, E. B. Fisher, and W. Lise, "Improved transmission representations in oligopolistic market models: Quadratic losses, phase shifters, and DC lines," In: IEEE Trans. Power Syst., vol. 23, no. 3, pp. 1018-1029, 2008.

[11] J. Barquin, E. Centeno, and J. Reneses, "Stochastic market equilibrium model for generation planning," In: Int. Conf. Probabilistic Methods Appl. Power Syst., pp. 367-372, 2004.

[12] C. Gao, T. Wu, Y. He, and R. Hu, "Generation and Transmission Coordinated Planning Considering Wind Power Integration," Automat. Elect. Power Syst., vol. 36, no. 22, pp. 30-35, 2012.

(C) Ming et al.; Licensee Bentham Open.

This is an open access article licensed under the terms of the Creative Commons Attribution Non-Commercial License (http://creativecommons.org/licenses/by$\mathrm{nc} / 4.0 /$ ) which permits unrestricted, non-commercial use, distribution and reproduction in any medium, provided the work is properly cited. 